\title{
A Influência das Edificações no Cálculo do Efeito Gravitacional das Massas Topográficas - Estudo de Caso na Cidade de Porto Alegre - RS (Brasil)
}

\section{The Influence of Buildings in Calculating the Gravitational Effect of Topographic Masses- A Case Study in the City of Porto Alegre - RS (Brazil)}

Rodrigo da Silva Ferraz ${ }^{1}$ e Sérgio Florêncio de Souza ${ }^{2}$

1 Universidade Federal do Rio Grande do Sul, Programa de Pós Graduação em Sensoriamento Remoto, Porto Alegre, Brasil. rferraz1980@gmail.com.

ORCID: https://orcid.org/0000-0003-1989-1424

2 Universidade Federal do Rio Grande do Sul, Programa de Pós Graduação em Sensoriamento Remoto, Porto Alegre, Brasil. sergioflorenciodesouza@gmail.com

ORCID: https://orcid.org/0000-0002-4720-6714

Resumo: Um dos objetivos da Geodésia consiste no estudo do geoide e sua determinação é obtida através do conhecimento do campo de gravidade que envolve a distribuição de massas na superfície terrestre. A abordagem clássica para a solução do problema de valor de contorno da Geodésia (PVCG) visando a determinação do geoide assume que os efeitos associados à topografia sejam levados em consideração. A técnica de Modelagem da Topografia Residual (RTM, em inglês Residual Terrain Modelling) tem como objetivo a modelagem o campo de gravidade em função da distribuição de massas associada a topografia onde, nesse tipo de estudo, o conteúdo de alta frequência do espectro relacionado a gravidade é gerado através desse método de redução associado a um modelo digital de elevação (MDE) de alta resolução. Nesse contexto, o objetivo principal desta pesquisa consiste em calcular o valor do potencial gravitacional das massas topográficas oriundas de edificações existentes na cidade de Porto Alegre - RS juntamente com a anomalia de gravidade associada à topografia. Esse estudo foi desenvolvido a partir de uma base vetorial com mais de 200 mil edificações onde o potencial gravitacional foi calculado a partir de um MDE gerado através de dados LiDAR (light detection and ranging). Para auxiliar nos cálculos, foi estimado um modelo de densidades em função das dimensões de cada edificação existentes na base de dados. Assim, foram calculados o valor do potencial gravitacional utilizando os elementos de massa tesseroide, prisma e massa pontual e também o valor da anomalia de gravidade para distâncias de $1 \mathrm{~km}, 2 \mathrm{~km}, 5 \mathrm{~km}, 10 \mathrm{~km}$ e $20 \mathrm{~km}$ usando a técnica de modelagem RTM. A influência das massas das edificações, neste estudo, representou 10,62\% do valor da anomalia de gravidade em comparação com o seu correspondente em relação ao solo.

Palavras-chave: Potencial gravitacional. Anomalia de gravidade. MDE. LiDAR. RTM.

Abstract: One of the goals of Geodesy is to study the geoid and its determination is obtained through knowledge of the gravity field that involves the distribution of masses on the Earth's surface. The classic approach to the solution of the geodetic boundary value problem (GBVP) aimed at determining the geoid assumes that the effects associated with the topography taken into account. The Residual Terrain Modelling (RTM) aims to model the gravity field as a function of the mass distribution associated with topography where, in this type of study, the high frequency content of the spectrum related to gravity is generated through this reduction method associated with a high resolution digital elevation model (DEM). In this contexto the main objective of this research is to calculate the value of the gravitational potential of the topographic masses from buildings in the city of Porto Alegre - RS together with the gravity anomalies associated with the topography. This study was developed from a vector base with more than 200 thousand buildings where the gravitational potential was calculated from an DEM generated through LiDAR (light detection and ranging) data. To assist in the calculations, a density model was estimated according to the dimensions of each building in the database. Thus, the value of the gravitational potential was calculated using the mass element tesseroid, prism and point mass and also the value of gravity for distances of $1 \mathrm{~km}, 2 \mathrm{~km}, 5 \mathrm{~km}, 10 \mathrm{~km}$ and $20 \mathrm{~km}$ using the RTM modeling technique. The influence of buildings masses, in this study, represented $10.62 \%$ of the value of the gravity anomalies compared to its corresponding in relation to the ground.

Keywords: Gravitational potential. Gravity anomalies. DEM. LiDAR. RTM. 


\section{INTRODUÇÃO}

Ao longo dos últimos anos, com o avanço da tecnologia, as técnicas de medição de coordenadas apresentaram uma grande evolução, possibilitando que informações tridimensionais fossem geradas a partir de estações totais, Fotogrametria, LiDAR (light detection and ranging) e posicionamento GNSS (Global Navigation Satellite System), de maneira rápida e eficaz. Entre os diversos métodos de medição de coordenadas, encontra-se o estudo das altitudes, um dos temas de estudo da geodésia, em que existem altitudes com características geométricas, geralmente associadas a observações GNSS, e outras com características físicas, como, por exemplo, a altitude ortométrica. No Brasil, alguns órgãos públicos utilizam a altitude ortométrica, tanto em sua base de dados vetorial, quanto na matricial, principalmente aqueles ligados ao governo federal que se seguem as Especificações Técnicas para Estruturação de Dados Geoespaciais Vetoriais - ET-EDGV. Assim, para se obter a altitude ortométrica a partir de dados altimétricos oriundos de posicionamento GNSS, necessita-se do valor da ondulação geoidal.

Um dos objetivos da Geodésia, além do estudo do geoide, consiste em determinar o campo de gravidade externo às massas, juntamente com a sua variação temporal e também a sua superfície limitante (GUIMARÃES; BLITZKOW, 2011). A geodésia utiliza a teoria do potencial, descrita por Laplace, como subsídio para o estudo do campo de gravidade e da forma geométrica da Terra, considerando que, fora das massas atrativas, o potencial obedece à equação de Laplace e, no interior das massas, ele segue a equação de Poisson. A soma do potencial gravitacional com o potencial centrífugo resulta no geopotencial, e este, juntamente com o esferopotencial, contribui para a determinação do potencial perturbador.

A teoria do potencial é aplicada no problema de valor de contorno da Geodésia (PVCG), na determinação do campo de gravidade externo às massas, e o cálculo do potencial gravitacional pode ser efetuado utilizando a técnica de modelagem da topografia residual (RTM, em inglês Residual Terrain Model) descrita por Forsberg (1984). A técnica de RTM é empregada para estimar o campo de gravidade por meio de um modelo digital de elevação (MDE) de alta resolução, valendo-se da integral de Newton para gerar os valores do potencial gravitacional (REXER et al., 2018). A integral de Newton pode ser discretizada analiticamente por elementos geométricos, como prismas ou tesseroides, e a estimativa do campo gravitacional é obtida pela soma dos efeitos de todos os elementos ao redor de um ponto de avaliação localizado na superfície topográfica (YANG; HIRT; PAIL, 2020).

Para o cálculo do potencial gravitacional, são utilizados prismas para aplicações locais em áreas com extensões mais reduzidas, onde as informações altimétricas geralmente estão relacionadas a coordenadas cartesianas (GROMBEIN; SEITZ; HECK, 2013). O uso de modelos esféricos, como, por exemplo, um tesseroide, requer um custo computacional um pouco maior devido à expansão harmônica esférica do potencial gravitacional derivado a partir da integral de Newton (NAGY; PAPP; BENEDEK, 2000). Esse tipo de modelagem também pode ser representado a partir de outros elementos geométricos como massa pontual, linha de massa e camada de massa (WILD-PFEIFFER, 2008) e por poliedros (SARASWATI et al., 2019).

As correções clássicas de terreno adotam aproximações que utilizam a eliminação das massas topográficas em função de um raio de integração. Em cálculos modernos de correção do terreno ou utilizando a técnica de modelagem RTM, como, por exemplo, os realizados por Tsoulis, Novák e Kadlec (2009), Kuhn e Hirt (2016) e Deng e Shen (2019), comumente são empregados os MDEs como base para estudos envolvendo a análise das massas topográficas, obtendo-se um melhor nível de precisão (HIRT et al., 2019).

Em alguns estudos, como os de Heck e Seitz (2007), Wild-Pfeifer (2008), Hirt et al. (2019) e Lin, Denker e Müller (2020), as análises referentes ao campo gravitacional acabam limitadas à superfície terrestre, e as massas residuais de elementos que se encontram acima da superfície terrestre, como, por exemplo, árvores, casas, prédios e demais edificações, acabam sendo negligenciadas em virtude da dificuldade de se estimar o valor de seu potencial. Uma forma de se obter a geometria desse tipo de feições, que servirão de base para o cálculo do potencial, consiste na medição de altitudes a partir de aerofotogrametria ou por dados LiDAR. A partir da Fotogrametria, dois produtos básicos são gerados: a ortofoto e o modelo digital de elevações (MDE) (MIKHAIL; BETHEL; MCGLONE, 2001). Através de processos matemáticos no MDE, pode-se chegar a um modelo digital de superfície (MDS) e a um modelo digital de terreno (MDT), e por meio de ambos pode-se estimar a altura da base do prédio, usando o MDT, e a altura do topo do prédio, usando o MDS. As dimensões 
dos prédios nos eixos X e Y podem ser geradas a partir de vetorização em algum software de SIG (Sistema de Informações Geográficas) por meio da ortofoto. A mesma analogia que foi adotada para a Fotogrametria pode ser utilizada para outros tipos de levantamentos como, por exemplo, o LiDAR. Esse tipo de levantamento gera um produto básico, uma nuvem de pontos com coordenadas tridimensionais que são medidas por intensidade de retorno de sinal, e os pulsos medidos podem ser separados por retornos - fato este que torna mais fácil e rápida a geração do MDT e do MDS, em comparação à Fotogrametria (VOSSELMAN; MASS, 2010).

Outro parâmetro que possui influência no cálculo do potencial gravitacional, a partir da integral de Newton, refere-se ao valor de densidade, e uma alternativa para estudos preliminares é a determinação de um valor médio a partir das dimensões de prédios. No presente estudo, esse valor foi obtido a partir de pesquisas feitas em revistas de construção civil, em que ele foi estimado em função da quantidade de massa empregada por metro quadrado e da altura de cada edificação.

Nesse contexto, o objetivo deste trabalho consiste em determinar o valor do potencial gravitacional associado à anomalia de gravidade a partir das massas das edificações existentes na cidade de Porto Alegre RS. Esta pesquisa foi desenvolvida usando uma base vetorial com mais de 200 mil edificações, tendo sido empregado um MDE gerado a partir de dados LiDAR. Por meio deste modelo de elevações foram geradas as alturas de todas as edificações dessa base vetorial, bem como um modelo de densidades para cada edificação em função da altura de cada prédio. Assim, foram realizados cinco experimentos e foram calculados os valores do potencial gravitacional utilizando os elementos de massa tesseroide, prisma e massa pontual para distâncias de $1 \mathrm{~km}, 2 \mathrm{~km}, 5 \mathrm{~km}, 10 \mathrm{~km}$ e $20 \mathrm{~km}$, juntamente com o cálculo da anomalia de gravidade associada à topografia.

\section{FUNDAMENTAÇÃO TEÓRICA}

Nesta seção serão abordados conceitos que estão diretamente envolvidos nas etapas desenvolvidas nesse estudo. Um dos objetivos da Geodésia, segundo Gemael (1999) e Heiskanen e Moritz (1967), consiste em determinar a forma da Terra bem como descrever a representação das variações do seu campo gravitacional.

A abordagem clássica para a solução do problema de valor de contorno da Geodésia para a determinação do geoide exige que os efeitos da topografia sejam levados em consideração (TZIAVOS; VERGOS; GRIGORIADIS, 2010). Isso se deve ao fato de Stokes, em 1849, assumiu que o potencial perturbador do campo de gravidade da Terra é harmônico fora do geoide, o que implica que todas as massas fora dessa superfície precisam ser removidas (HEISKANEN; MORITZ, 1967). De um modo geral, a atração gravitacional de massas topográficas cria um forte sinal de que domina o espectro de gravidade em comprimentos de onda mais curtos e, portanto, a topografia pode ser usada para suavizar o campo de gravidade antes de qualquer processo de modelagem, ou seja, os ruídos inerentes ao campo de gravidade podem ser suavizados pelas reduções de terreno (TZIAVOS; SIDERIS, 2013).

Nesse contexto, se insere a técnica de RTM que pode ser considerada como um caso de modelagem do campo gravitacional gerado por alguma distribuição de massa topográfica. Através da aplicação da integral de Newton, essa técnica de modelagem fornece o campo de gravidade associado à topografia. Por outro lado, ela produz apenas os constituintes do campo gravitacional referentes aos sinais dos curtos comprimentos de onda. Para solucionar esse problema são utilizados MDE de alta resolução com a finalidade de obter os dados referentes aos longos comprimentos de onda com o objetivo de modelar o campo gravitacional gerado pelas distribuições de massa relacionados à topografia (HIRT et al., 2019). Segundo Schwarz (1984), entre 2 e 34\% dos espectros relacionados à anomalia de altitude e efeitos relacionados ao campo de gravidade associados ao cálculo do geoide estão contidos nas altas frequências onde a utilização de MDE de alta resolução desempenham um papel significativo (TZIAVOS; SIDERIS, 2013). A integral de Newton, utilizada na técnica de modelagem RTM, pode ser decomposta analiticamente em elementos geométricos denominados elementos de massa, como um tesseroide, prisma, massa pontual, camada de massa e linha de massa (WILD-PFEIFFER, 2008). Uma das representações do efeito do potencial gravitacional, pode ser descrito pela integral de Newton em coordenadas esféricas conforme a Eq. (1) (GROMBEIN; SEITZ; HECK, 2013) 


$$
V=G \iiint_{\Omega} \frac{\rho}{l} d \Omega
$$

onde $V$ representa o valor do potencial gravitacional, $G$ a constante de gravitação universal de Newton (6,67 $\cdot 10^{-11} \mathrm{~m}^{3} / \mathrm{kg} \cdot \mathrm{s}^{2}$ ), $\rho$ representa o valor de densidade, $l$ a distância euclidiana entre as massas e o elemento $d \Omega$ representa os elementos infinitesimais da integral de volume. A integral de Newton descrita na Eq. (1) pode ser desenvolvida analiticamente através de elementos geométricos como tesseroides, prismas e massa pontual.

O valor da anomalia de gravidade do RTM é descrito pela Eq. (2) (FORSBERG, 1984; TZIAVOS; SIDERIS, 2013).

$$
\Delta \mathrm{g}_{\mathrm{RTM}}=2 \pi \mathrm{G} \rho\left(\mathrm{H}-\mathrm{H}_{\mathrm{ref}}\right)-\mathrm{G} \iint_{E} \int_{H_{r e f}}^{H} \rho \frac{H_{p}-z}{l^{3}} d x d y d z
$$

Onde $G$ representa a constante de gravitação universal de Newton, $\rho$ representa o valor da densidade adotada (geralmente $2670 \mathrm{~kg} / \mathrm{m}^{3}$ ), $H$ a origem do ponto de cálculo, $H_{\text {ref }}$ a altura da feição que será calculado o efeito gravitacional, $\rho$ o valor da densidade e $l$ a distância euclidiana do ponto de cálculo até o elemento analisado.

A integral de Newton pode ser discretizada através de um tesseroide, Figura 1, é um elemento geométrico delimitado por duas esferas concêntricas com raios $\mathrm{r} 1$ e $\mathrm{r} 2$ constantes, dois meridianos planos ( $\lambda 1$ $=$ constante, $\lambda 2=$ constante) e duas superfícies cônicas cujas linhas de origem passam pelo centro da esfera e ao longo dos paralelos $\varphi 1=$ constante, $\varphi 2=$ constante (HECK; SEITZ, 2007).

Figura 1 - Representação de um tesseroide.

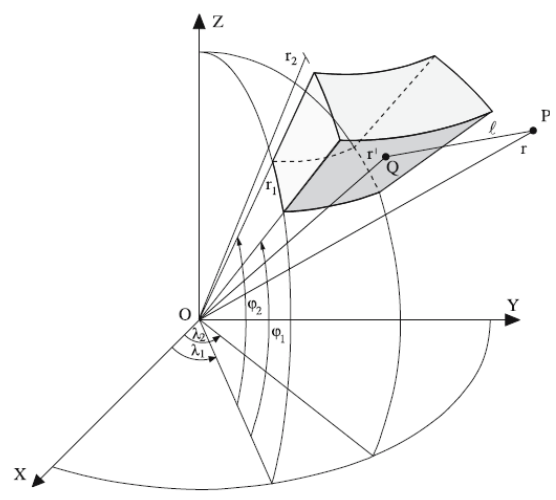

Fonte: Heck e Seitz (2007).

O valor da distância da origem do sistema até o centroide do polígono $(l)$ que representa o tesseroide é representado pela Eq. (3) e Eq. (4):

$$
\begin{gathered}
\cos \psi_{0}=\sin \varphi \cdot \sin \varphi_{0}+\cos \varphi \cdot \cos \varphi_{0} \cdot \cos \left(\lambda-\lambda_{0}\right) \\
l_{0}=\sqrt{r^{2}+r_{0}^{2}-2 \cdot r \cdot r_{0} \cdot \cos \psi_{0}}
\end{gathered}
$$

O valor do potencial gravitacional $V(r, \varphi, \lambda)$ é determinado a partir das Eq. (5) a Eq. (9). Nota-se que na Eq. (5) os efeitos da altura do tesseroide $(\Delta r)$ começam a ser considerados bem como o efeito da densidade ( $\rho)$ da superfície.

$$
\begin{gathered}
\Delta \mathrm{r}=\mathrm{r} 2-\mathrm{r} 1=\mathrm{h} 2-\mathrm{h} 1 \\
\Delta \varphi=\varphi 2-\varphi 1
\end{gathered}
$$




$$
\begin{gathered}
\Delta \lambda=\lambda 2-\lambda 1 \\
\mathrm{~L}_{000}=\frac{\mathrm{r}^{2} \cdot\left(\mathrm{r}^{\prime}-\mathrm{r} \cdot \cos \psi_{0}\right) \cdot \cos \varphi_{0}}{\mathrm{l}^{3}} \\
\mathrm{~V}(\mathrm{r}, \varphi, \lambda)=\mathrm{G} \rho \Delta \mathrm{r} \Delta \varphi \Delta \lambda \mathrm{L}_{000}\left[1+\mathrm{O}\left(\Delta^{2}\right)\right]
\end{gathered}
$$

O potencial gravitacional também pode ser descrito por um prisma retangular e homogêneo, conforme a Figura 2, que pode ser descrito analiticamente pela integral de Newton em coordenadas planas através das Eq. (10) e Eq. (11) (NAGY; PAPP; BENEDEK, 2000).

$$
\begin{aligned}
& \mathrm{V}(\mathrm{x}, \mathrm{y}, \mathrm{z})=\mathrm{G} \rho \int_{\mathrm{z} 1}^{\mathrm{z} 2} \int_{\mathrm{y} 1}^{\mathrm{y} 2} \int_{\mathrm{x} 1}^{\mathrm{x} 2} \frac{\mathrm{dx}^{\prime} d \mathrm{y}^{\prime} \mathrm{dz}}{\mathrm{l}} \\
& l=\sqrt{\left(x-x^{\prime}\right)^{2}+\left(y-y^{\prime}\right)^{2}+\left(z-z^{\prime}\right)^{2}}
\end{aligned}
$$

Figura 2 - Geometria de um prisma retangular.

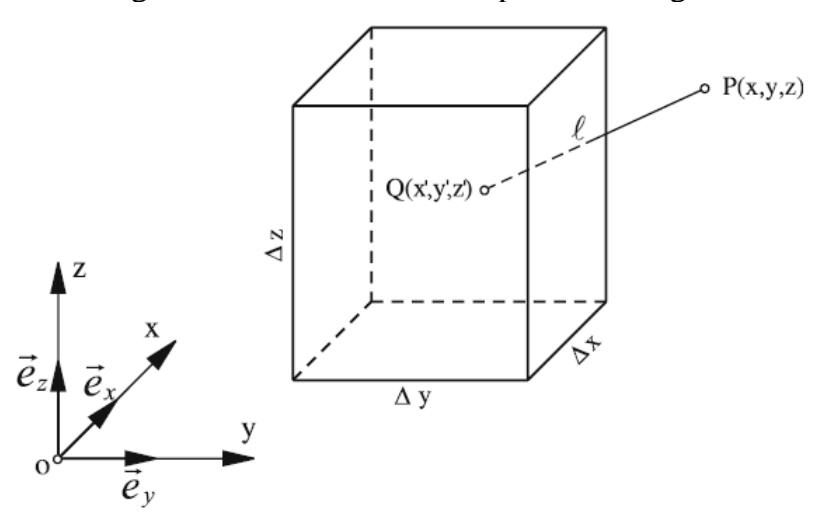

Fonte: Heck e Seitz (2007).

A integral descrita na Eq. (10) pode ser resolvida analiticamente resultando no valor do potencial $V(x, y, z)$ descrito na Eq. (12).

$$
\begin{gathered}
V(x, y, z)=G \rho \sum_{i=1}^{2} \sum_{j=1}^{2} \sum_{k=1}^{2}(-1)^{i+j+k} \\
\times\left[\left(x-x_{i}\right)\left(y-y_{j}\right) \ln \left|\frac{z-z_{k}+w_{i j k}}{\sqrt{\left(x-x_{i}\right)^{2}+\left(y-y_{j}\right)^{2}}}\right|\right. \\
+\left(y-y_{j}\right)\left(z-z_{k}\right) \ln \left|\frac{x-x_{i}+w_{i j k}}{\sqrt{\left(y-y_{j}\right)^{2}+\left(z-z_{k}\right)^{2}}}\right| \\
+\left(z-z_{k}\right)\left(x-x_{i}\right) \ln \left|\frac{y-y_{k}+w_{i j k}}{\sqrt{\left(z-z_{k}\right)^{2}+\left(x-x_{i}\right)^{2}}}\right| \\
-\frac{1}{2}\left(\left(x-x_{i}\right)^{2} \arctan \frac{\left(y-y_{j}\right)\left(z-z_{k}\right)}{\left(x-x_{i}\right) w_{i j k}}\right. \\
+\left(y-y_{j}\right)^{2} \arctan \frac{\left(z-z_{k}\right)\left(x-x_{i}\right)}{\left(y-y_{j}\right) w_{i j k}}
\end{gathered}
$$




$$
+\left(\mathrm{z}-z_{k}\right)^{2} \arctan \frac{\left(\mathrm{x}-x_{i}\right)\left(\mathrm{y}-y_{j}\right)}{\left(\mathrm{z}-z_{k}\right) \mathrm{w}_{\mathrm{ijk}}}
$$

Outra forma de descrever o potencial gravitacional pode ser representado pelo elemento massa pontual , cuja formulação matemática é descrita por Heck e Seitz (2007), Wild-Pfeifer (2008), Grombein, Seitz e Heck (2013) e Marotta et al. (2019), onde o valor da massa total do prisma fica concentrado no centro geométrico da Figura 3 e o valor do potencial $V(x, y, z)$ é descrito pela Eq. (13).

Figura 3 - Representação do elemento massa pontual em um prisma retangular.

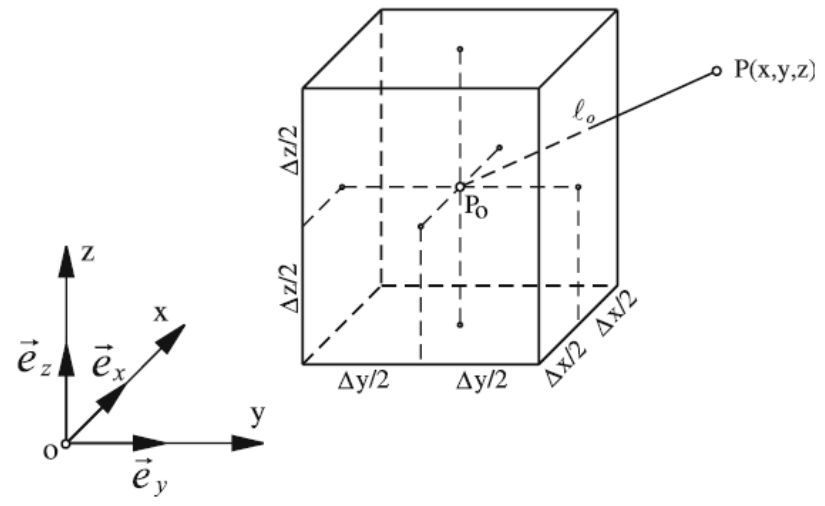

Fonte: Heck e Seitz (2007).

$$
V(\mathrm{x}, \mathrm{y}, \mathrm{z})=\frac{\mathrm{G} \rho \Delta \mathrm{x} \Delta \mathrm{y} \Delta \mathrm{z}}{\mathrm{l}_{\mathrm{o}}}\left[1+\mathrm{O}\left(\Delta^{2}\right)\right]
$$

Em relação a estudos envolvendo o cálculo do valor do potencial gravitacional existem alguns trabalhos que abordam esse tema como por exemplo Heck e Seitz (2007), Wild-Pfeifer (2008) onde os mesmos adotam modelos com densidade $(\rho)$ e alturas dos prismas com valores constantes. Li et al. (2011) adota um modelo de densidades com valores que apresentam uma pequena variação, mas a nível da estrutura do solo, sem considerar a influência das massas de edificações que se encontram acima dessa superfície. Seguindo a mesma linha de pesquisa existem estudos como os desenvolvidos por Deng e Shen (2009) na China, Tsoulis, Novàk e Kadlec (2009) na Europa central, Yahaya e El Azzab (2018) na Nigéria, Varga et al. (2019) na Croacia e Hirt et al. (2019) no Himalaia.

Uma aplicação da modelagem RTM pode ser descrita nos estudos realizados Omang e Forsberg (2000), numa região entre a Finlândia e a Dinamarca onde os autores obtiveram um erro no valor do cálculo do geoide em torno de $5 \mathrm{~cm}$. Um estudo semelhante também foi realizado por Tziavos, Vergos e Grigoriadis (2010) para estimar o valor do geoide, na Grécia, adotando diferentes MDE os autores obtiveram erros entre 3,3 e 14,0 mGal nas anomalias do campo gravitacional e entre 1,6 a 26,0 cm no valor do geoide.

Verificou-se que em todos os trabalhos citados anteriormente os valores de densidades são adotados como constantes ou não são o tema principal dos referidos trabalhos e um estudo que aborda a utilização de valores de densidades utilizando modelos de massa foi o desenvolvido no Distrito Federal do Brasil por Marotta, Almeida e Cherubim (2019) na estimativa de um modelo geoidal local.

Uma breve abordagem sobre a tecnologia LiDAR também será descrita uma vez que os modelos digitais de elevação utilizados para se medir a base e o topo dos prédios, que foram usados nesse estudo, foram obtidos através dessa técnica. O LiDAR consiste em um sensor que mede coordenadas tridimensionais a partir de emissão de pulsos de energia que são enviados diretamento em direção ao alvo com um determinado ângulo em relação ao espelho do sensor. Dependendo da posição do instrumento, cada pulso irá iluminar no alvo uma área com um formato de um círculo, denominada de área de cobertura laser instantânea. Esse pulso será refletido e pode gerar um retorno ou múltiplos retornos e cada um deles é registrado no sensor como uma coordenada (SHAN; TOTH, 2008).

Assim, praticamente toda a energia emitida pelo sensor interage com a superfície do terreno e seria assumido que essa interação resultasse num único retorno. Entretando as diferentes feições que compõem o 
terreno irão gerar diferentes retornos em função da forma e dimensão do alvo que receberá o pulso incidente. Desta forma, cada pulso transmitido da fonte emissora pode gerar múltiplos retornos (VOSSELMAN; MAAS, 2010). Após o processamento dos dados e a separação dos retornos os modelos digitais que descrevem o terreno podem ser gerados. Os retornos intermediários irão gerar o modelo digital de superfície (MDS) e os últimos retornos irão gerar os modelos digitais de terreno (MDT).

Nesse contexto, em 2008 a Prefeitura Municipal de Porto Alegre (PMPA) fez um voo fotogramétrico onde os dados altimétricos foram gerados por levantamento LiDAR com a finalidade de atualização da Planta Genérica de Valores (PGV) da cidade. A partir desse levantamento praticamente todas as edificações da cidade foram digitalizadas em ambiente SIG onde foi gerada uma base vetorial, para esse estudo, com mais de 200 (duzentas) mil edificações.

Os voos para execução do levantamento LiDAR foram efetuados em agosto de 2010, com 68 faixas na direção Norte-Sul e com superposição lateral de 30\%. Os parâmetros de voo e configuração dos scanners foram definidos de forma a proporcionar uma densidade mínima de 2,2 pontos por $\mathrm{m}^{2}$. Foram utilizados os scanners ALS50 e ALS60, ambos da LEICA Geosystems. Para a validação da altimetria foram medidos 92 pontos de controle chegando a um valor médio de discrepâncias de 7,5 cm com desvio-padrão de 6,0 centímetros. Para maiores detalhes sobre as especificações desse levantamento pode se consultar Zanardi et al. (2013).

\section{EXPERIMENTOS REALIZADOS}

Os estudos foram desenvolvidos na cidade de Porto Alegre, no Estado do Rio Grande do Sul, Brasil, conforme a Figura 4. A cidade de Porto Alegre, que é a capital do Estado, possui uma área de aproximadamente $500 \mathrm{~km}^{2}$. As distâncias entre os eixos norte e sul são de aproximadamente $34 \mathrm{~km}$ e de $18 \mathrm{~km}$ no eixo Leste/Oeste, com uma altitude média de 10 metros acima do nível do mar.

Figura 4 - Mapas de localização da área de estudo.

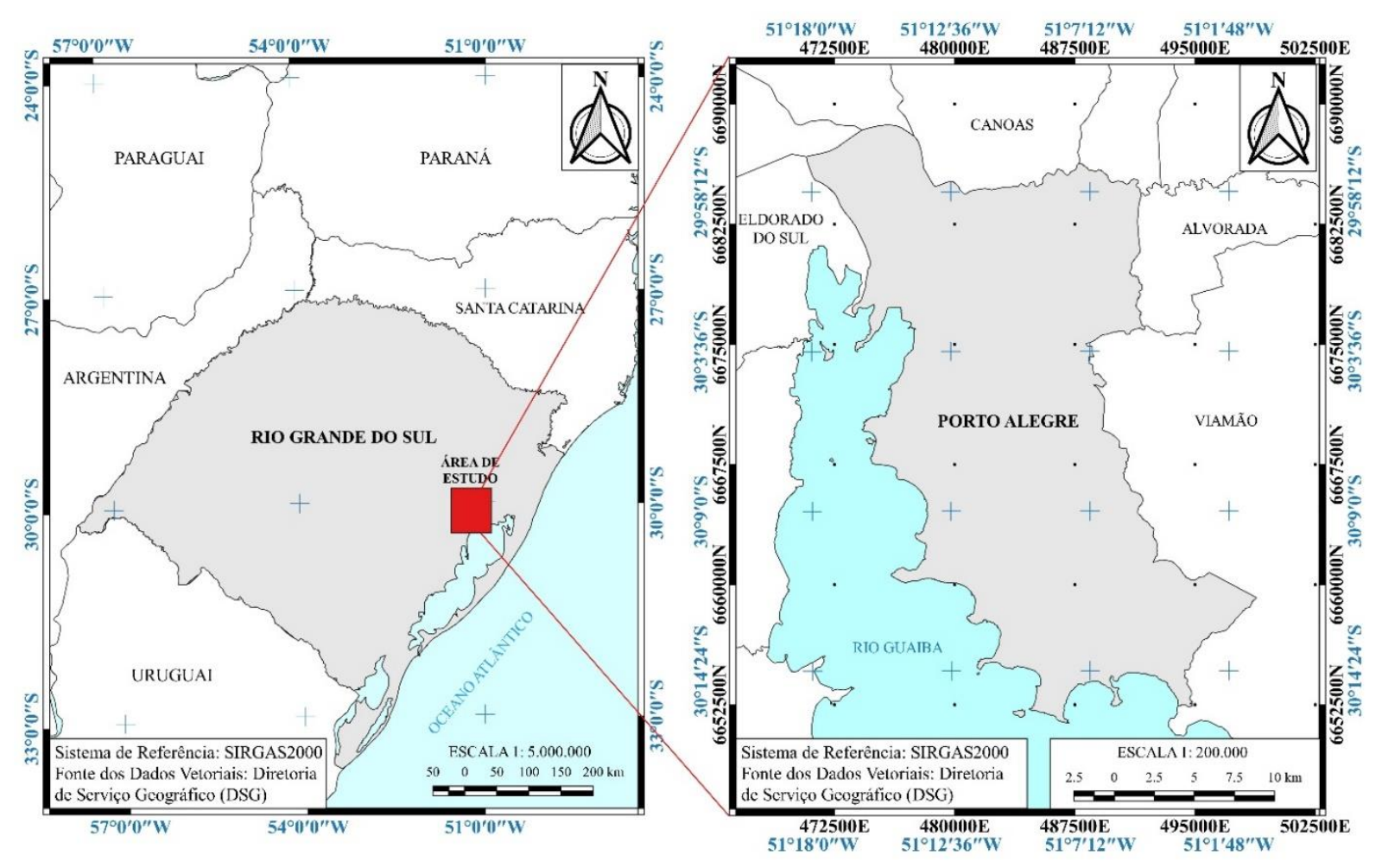

Fonte: Os autores (2021).

Segundo a Prefeitura Municipal de Porto Alegre (PMPA), os prédios mais altos são os edifícios Santa Cruz, com 32 andares e 96 metros de altura, o Coliseu, com 28 andares e 84 metros de altura, e o Malcon, com 27 andares e 81 metros de altura. A partir dessas informações, verificou-se que a altura de cada andar ou nível dos referidos edifícios corresponde a aproximadamente três metros e adotou-se esse parâmetro como altura 
mínima de cada edificação. Assim, para diminuir o custo computacional, foram mantidos na base apenas aqueles dados referentes a edificações com pelo menos três metros de altura.

Após a descrição do local dos experimentos (Figura 4), a Figura 5 procura sintetizar um resumo de todas as atividades desenvolvidas neste estudo. Inicialmente foi criada uma base de dados de trabalho a partir dos vetores das edificações oriundos da PMPA. Após essa etapa, utilizando as nuvens de pontos dos dados LiDAR, gerou-se um MDS e um MDT com resolução espacial de dois metros da área da cidade de Porto Alegre. A partir da diferença do MDS e MDT, obteve-se a estimativa de altura dos prédios onde se efetuou uma filtragem nas edificações, deixando apenas aquelas edificações com alturas superiores a 3 (três) metros.

Figura 5 - Fluxograma das atividades desenvolvidas no estudo.

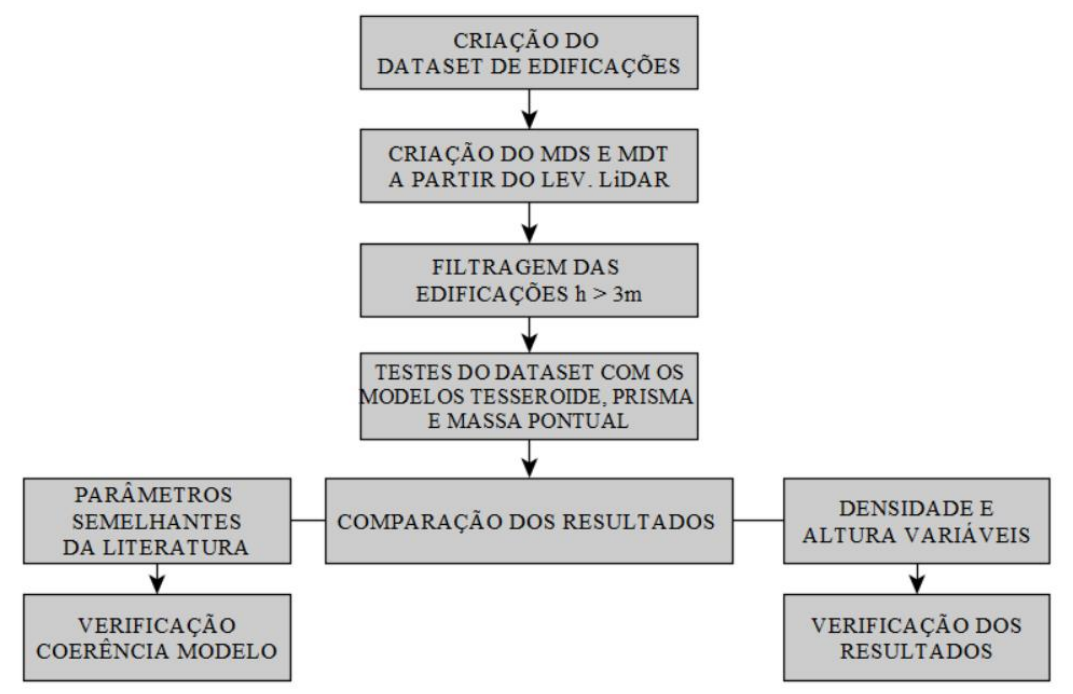

Fonte: Os autores (2021).

Após o processo de filtragem das edificações foi aplicada a técnica de RTM usando os elementos de massa tesseroide, prisma e massa pontual na base de dados e na sequência os dados gerados foram comparados, dentro das suas proporções, com o estudo desenvolvido por Heck e Seitz (2007) visando verificar a coerência dos valores calculados. Nesse estudo, foi utilizado como MDE de referência o MDT gerado pelos dados LiDAR. Os dados altimétricos utilizados para o cálculo da anomalia de gravidade associada as massas das edificações foram gerados a partir do MDS oriundo dos dados LiDAR.

Em relação ao modelo de densidades, utilizou-se o padrão descrito em uma reportagem do jornal The Seattle Times (2004), que descreve uma distribuição de massas de $984,782 \mathrm{~kg} / \mathrm{m}^{2}$ para edificações de até um andar, de $1.354,076 \mathrm{~kg} / \mathrm{m}^{2}$ para edificações com dois andares e de $1.723,05 \mathrm{~kg} / \mathrm{m}^{2}$ para edificações de três andares. Neste estudo, adotou-se o padrão do valor de três andares para edificações com mais de três andares, conforme a Tabela 1.

Tabela 1 - Modelo de distribuição de massas adotado nesse estudo.

\begin{tabular}{c|c}
\hline Andares/Níveis & Massa Empregada \\
\hline 01 Andar ou Nível & $984,782 \mathrm{~kg} / \mathrm{m}^{2}$ \\
02 Andares ou Níveis & $1.354,076 \mathrm{~kg} / \mathrm{m}^{2}$ \\
A partir de 03 andares & $1.723,05 \mathrm{~kg} / \mathrm{m}^{2}$ \\
\hline
\end{tabular}

Fonte: Os autores (2021).

Para a obtenção do modelo de densidades de cada edificação, estimou-se:

1. A quantidade de níveis ou andares de cada edificação. Obteve-se a altura de cada prédio pela diferença entre os valores do MDS e do MDT, oriundos do levantamento LiDAR. A quantidade de níveis de cada edificação foi estabelecida pela divisão da altura de cada prédio por três metros. Assumiu-se o valor inteiro.

2. A massa empregada de cada prédio em kg. Esta foi obtida por meio da multiplicação da área de cada prédio pela quantidade de níveis. Em seguida, esses valores foram multiplicados pelos seus 
correspondentes na Tabela 1.

3. O volume de cada edifício em $\mathrm{m}^{3}$. Multiplicou-se a área de cada edificação por sua respectiva altura.

4. Finalmente, o valor de densidade de cada edifício em $\mathrm{kg} / \mathrm{m}^{3}$. Dividiu-se a estimativa da massa de cada edificação pelo seu respectivo volume.

Assim, cada edificação da base de dados teve um valor de densidade diferente, sendo o valor mínimo de $187,611 \mathrm{~kg} / \mathrm{m}^{3}$ e o valor máximo de $590,764 \mathrm{~kg} / \mathrm{m}^{3}$.

Figura 6 - Distribuição das edificações nos experimentos realizados.

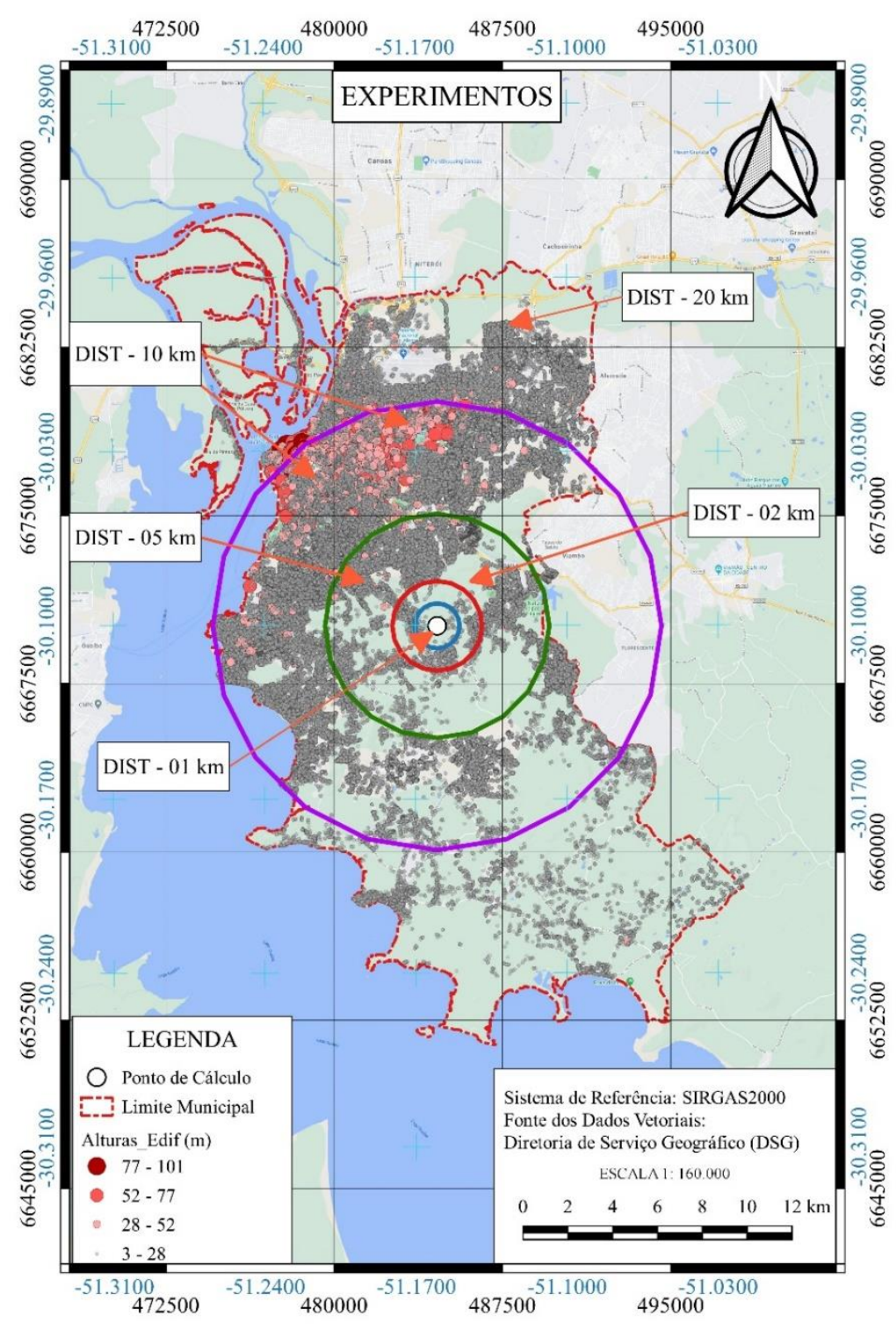

Fonte: Os autores (2021).

A Figura 6 é um mapa da cidade de Porto Alegre em escala 1:160.000. Na mesma estão representados os raios de avaliação de $01 \mathrm{~km}, 02 \mathrm{~km}, 05 \mathrm{~km}, 10 \mathrm{~km}$ e $20 \mathrm{~km}$ referentes aos cinco experimentos. O ponto de origem, para o cálculo do potencial gravitacional, fica localizado entre os bairros Cascata e Belém Velho, com uma altitude de 12,822 m. É possível também observar nessa figura que as edificações mais altas encontramse nos experimentos com distâncias de 10 e $20 \mathrm{~km}$.

Os cálculos empregados nas Eq. (1) à Eq. (13) foram implementados na linguagem de programação Python versão 3.7. Foram utilizados os módulos Numpy e Tensorflow para as operações matriciais, Pandas para o tratamento de bases de dados e Matplotlib para a geração de gráficos. Utilizou-se dois poderosos ambientes científicos como o IDE (Integrated Development Environment) Spyder versão 4.0.1 e o Google Colaboratory (Google Colab). 


\section{ANÁLISE DOS RESULTADOS}

Esta seção aborda os resultados referentes ao cálculo do valor de $V$ por meio da modelagem RTM, empregando os elementos de massa tesseroide, prisma e massa pontual e o cálculo do efeito gravitacional gerado por esses elementos. Na Tabela 2 consta um resumo de todos os testes realizados neste trabalho. Na coluna "Experimento" consta as distâncias usadas nos testes, e na coluna "N. ${ }^{\circ}$ Prédios" consta a quantidade de prédios existentes em cada experimento. Também foram inseridos na Tabela 2 os valores de alturas (“Alt.") mínimas e máximas em cada experimento, bem como o valor médio do potencial gravitacional $V$ associado a cada elemento de massa, no caso o tesseroide, prisma e massa pontual. Os valores de $V$ apresentaram resultados bastante semelhantes, ou seja, o valor médio para edificações a uma distância de $1 \mathrm{~km}$ variou entre $0,024 \mathrm{~m}^{2} / \mathrm{s}^{2}$, com elemento de massa tesseroide, e o valor de $0,027 \mathrm{~m}^{2} / \mathrm{s}^{2}$, com os elementos de massa prisma. Essa pequena diferença também ocorreu nos experimentos com distâncias de $2 \mathrm{~km}, 5 \mathrm{~km}, 10 \mathrm{~km}$ e $20 \mathrm{~km}$. Estima-se que houve pouca variação nesses valores em função de as distâncias dos experimentos não serem superiores a 20 $\mathrm{km}$, uma vez que nos experimentos realizados por Heck e Seitz (2007) esses valores começaram a ficar evidentes a partir da distância de $9 \mathrm{~km}$.

Tabela 2 - Experimentos realizados.

\begin{tabular}{c|c|c|c|c|c|c}
\hline \multirow{2}{*}{ Experimento } & $\mathbf{N}^{\mathbf{0}}$ Prédios & $\begin{array}{c}\text { Alt Prédios }- \\
\mathbf{M i n}(\mathbf{m})\end{array}$ & $\begin{array}{c}\text { Alt Prédios }- \\
\mathbf{M a x}(\mathbf{m})\end{array}$ & $\begin{array}{c}V \text { Tesseroide } \\
\left(\mathbf{m}^{2} / \mathbf{s}^{2}\right)\end{array}$ & $\begin{array}{c}V \text { Prisma } \\
\left(\mathbf{m}^{2} / \mathbf{s}^{2}\right)\end{array}$ & $\begin{array}{c}V \text { Massa Pontual } \\
\left(\mathbf{m}^{2} / \mathbf{s}^{2}\right)\end{array}$ \\
\hline $01 \mathrm{~km}$ & 313 & 3,009 & 15,388 & 0,024 & 0,027 & 0,025 \\
$02 \mathrm{~km}$ & 3742 & 3,000 & 19,542 & 0,071 & 0,070 & 0,070 \\
$05 \mathrm{~km}$ & 36881 & 3,000 & 45,392 & 0,167 & 0,162 & 0,160 \\
$10 \mathrm{~km}$ & 150375 & 3,000 & 94,143 & 0,403 & 0,400 & 0,403 \\
$20 \mathrm{~km}$ & 58173 & 3,000 & 101,400 & 0,487 & 0,485 & 0,487 \\
\hline
\end{tabular}

Fonte: Os autores (2021).

$\mathrm{Na}$ integral de Newton, descrita na Eq. (1), os dois principais parâmetros envolvidos no cálculo do valor de $V$ (sem contar a constante de gravitação universal) são o valor da densidade $\rho$ e a distância ao ponto de cálculo do sistema. A altura dos prédios foi um dos parâmetros utilizados para estimar o modelo de densidades de cada edificação e verificou-se que os maiores valores de $V$ foram encontrados nos experimentos que continham as edificações com maiores valores de altura.

Figura 7 - Valor do potencial gravitacional $V$ em função da densidade e da altura das edificações.
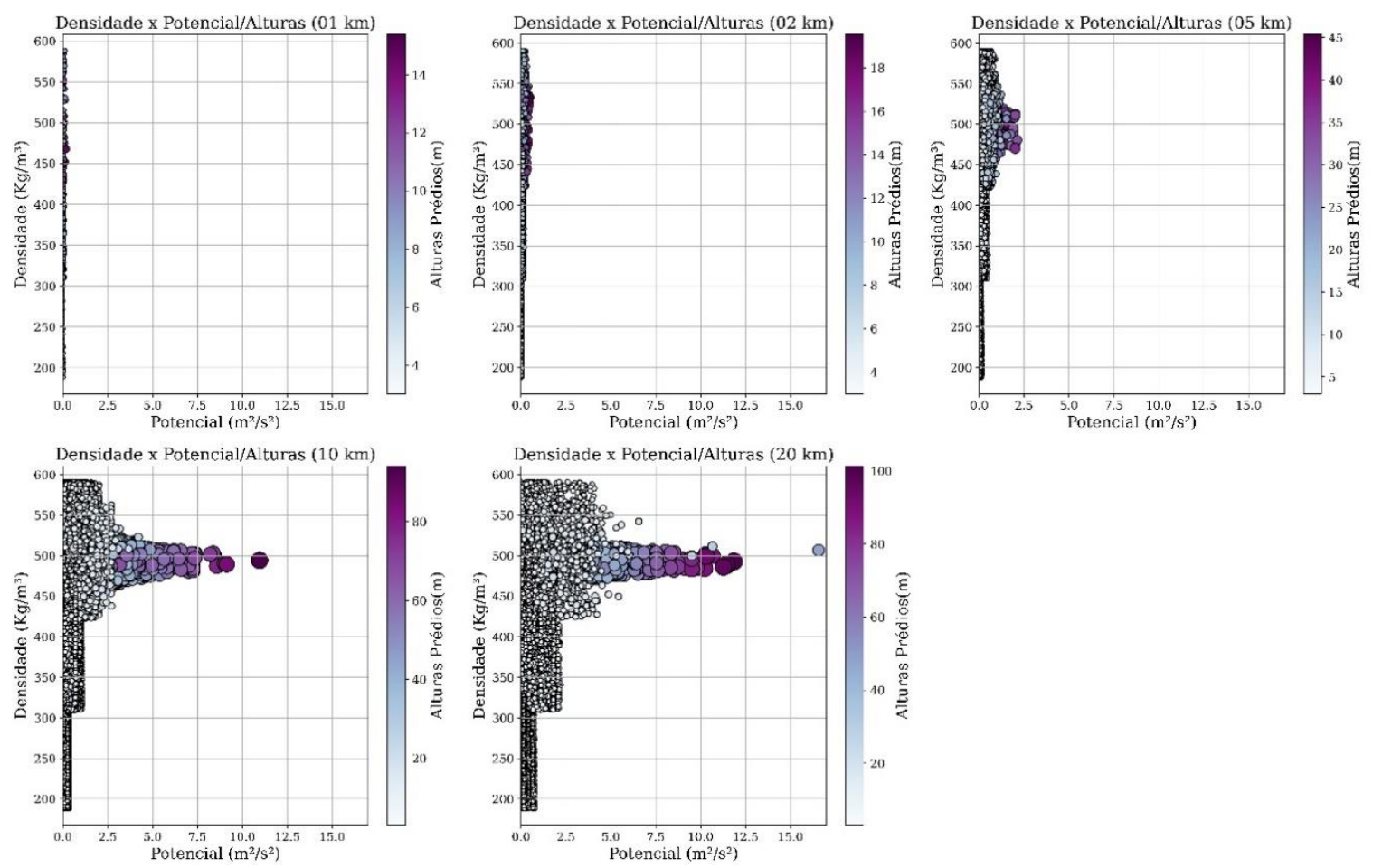

Fonte: Os autores (2021). 
Os maiores valores de $\mathrm{V}$ foram obtidos nos experimentos com distâncias de $10 \mathrm{~km}$ e $20 \mathrm{~km}$, sendo que ambos foram influenciados diretamente pela quantidade de prédios (208.548 ao total), evidenciando que essa variável possui uma relação direta com o valor de V. Assim, na Figura 7, procurou-se representar a relação existente entre o valor de V em função da densidade e da altura dos prédios. Na Figura 7, a quantidade de círculos representa o número de edificações em cada experimento, o diâmetro de cada círculo representa as alturas de cada prédio e os círculos de cores mais escuras representam as edificações mais altas. Constatou-se que os maiores valores de $\mathrm{V}$ foram gerados nas faixas de densidade entre $400 \mathrm{~kg} / \mathrm{m}^{3}$ e $550 \mathrm{~kg} / \mathrm{m}^{3}$ e com alturas entre $60 \mathrm{~m}$ e $80 \mathrm{~m}$.

Portanto, procurou-se verificar se existia algum tipo de relação matemática (linear, quadrática ou exponencial) entre o valor de $V$ e as distâncias entre os elementos de massa analisados. Como pode ser verificado na Figura 8, os experimentos apresentaram um comportamento linear entre o valor do potencial com o incremento da distância, onde o maior valor foi verificado no experimento com distância de $20 \mathrm{~km}$.

Figura 8 - Comparação dos resultados em cada experimento.

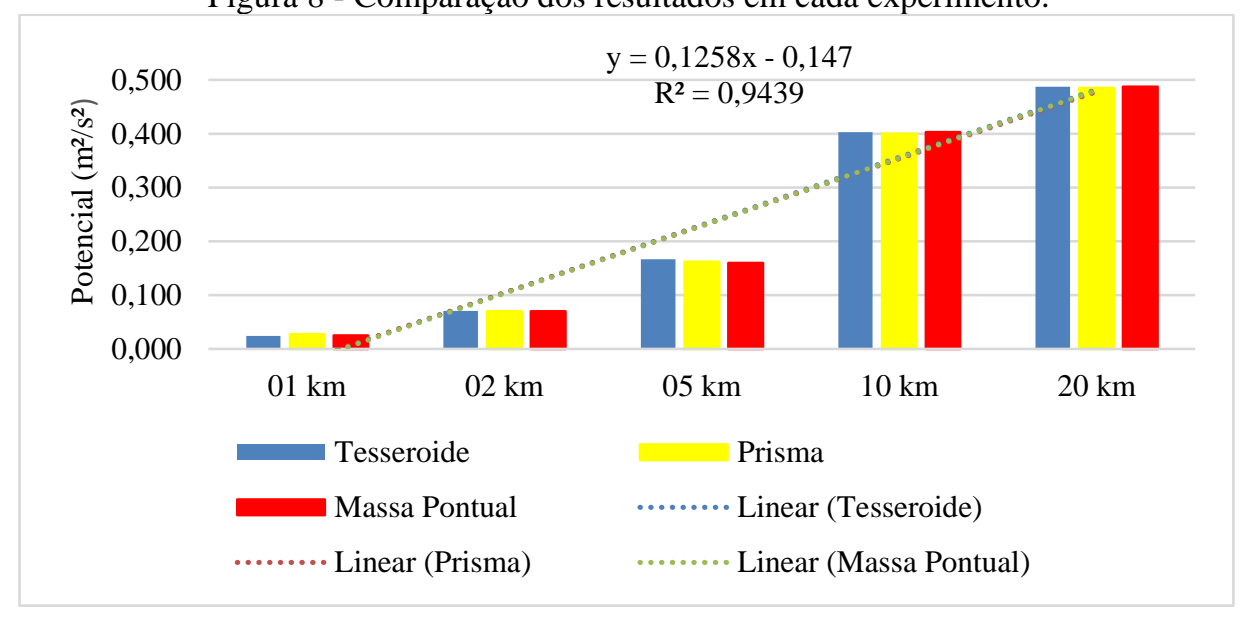

Fonte: Os autores (2021).

Assim, para verificar se houve diferença significativa entre os experimentos foi realizado um teste de análise de variância (ANOVA). Este é um teste de hipótese não paramétrico onde a hipótese básica $\left(\mathrm{H}_{0}\right)$ indica que não existe variação significativa entre os dados analisados dentro do mesmo grupo e entre os outros grupos de comparação e a hipótese alternativa $\left(\mathrm{H}_{1}\right)$ indica que os dados possuem variância significativa. (MONTGOMERY; RUNGER; CALADO, 2000). Nesse caso, as hipóteses testadas foram:

- $\mathrm{H}_{0}$, hipótese básica, indica que não existe variação significativa entre os dados analisados dentro do mesmo grupo e entre outros grupos de comparação. Os grupos de comparação representam os valores de $V$ utilizando os elementos de massa tesseroide, prisma e massa pontual.

- $\mathrm{H}_{1}$, hipótese alternativa, indica que existe diferença significativa entre os experimentos.

$\mathrm{Na}$ Tabela 3 consta os resultados do teste ANOVA. Comparando o valor de $\mathrm{F}_{\text {Calculado, }}$ que corresponde a quociente entre a variação entre os grupos e a variação dentro dos grupos, com o valor de $\mathrm{F}_{\text {Teórico }}$ (tabelado) nota-se que ele é inferior ao valor teórico aceitando-se assim a hipótese básica a um nível de significância de 95\%. Outra maneira de se avaliar a significância da estatística do teste ANOVA consiste na análise do valor$\mathrm{P}$. Caso valor-P $<\alpha$ ou valor-P $<0,05$ rejeitamos a hipótese básica, caso contrário, não se rejeita a hipótese de nulidade de $\mathrm{H}_{0}$, ou seja, não há evidências de diferenças significativas entre os experimentos. Conforme o exemplo desse estudo, descrito na Tabela 3 , o valor-P > 0,05 ratifica a aceitação de $\mathrm{H}_{0}$ e a rejeição de $\mathrm{H}_{1}$.

Tabela 3 - Análise de variância (ANOVA) entre os experimentos.

\begin{tabular}{l|c|c|c|c|c|c}
\hline \multicolumn{1}{c|}{ Fonte da variação } & SQ & GL & MQ & F $_{\text {Calculado }}$ & valor-P & F \\
\hline Entre gruposico & 0,002 & 0,998 & 3,885 \\
Dentro dos grupos & 0,000 & 2,000 & 0,000 & & & \\
Total & 0,070 & 12,000 & 0,006 & & & \\
\hline
\end{tabular}

Fonte: Os autores (2021). 
Logo, a partir dos dados obtidos na Tabela 3, nota-se que não existem diferenças significativas entre os experimentos onde o valor-P é de 0,998>0,05 rejeitando a hipótese alternativa e aceitando a hipótese básica, ou seja, nesse estudo a utilização dos elementos de massa tesseroide, prisma e massa pontual não apresentaram valores com diferenças significativas para distâncias de 01 km, 02 km, 05 km, 10 km e 20 km.

A integral de Newton, Eq. (1), considera a densidade e distância do ponto de cálculo até o elemento de massa como parâmetros fundamentais. Pelo gráfico da Figura 8 notou-se que o valor do potencial apresentou uma relação linear em cada experimento, assim, estimou-se esse tipo de relação entre a distância ao sistema de origem e também em relação as variáveis que foram utilizadas para se chegar ao valor da densidade de cada edificação, no caso a quantidade de prédios e a altura de cada prédio, através de uma análise de correlação conforme a Figura 9.

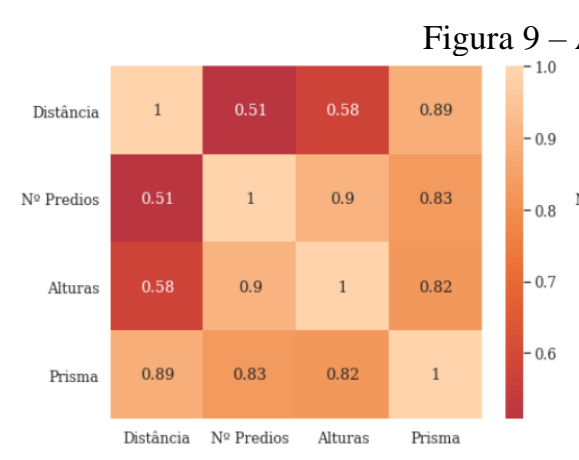

(a)

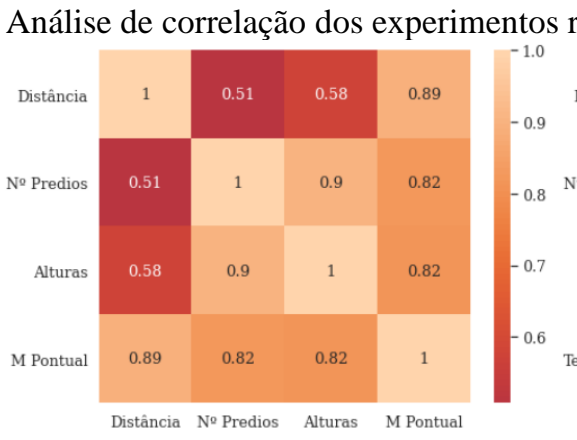

(b)

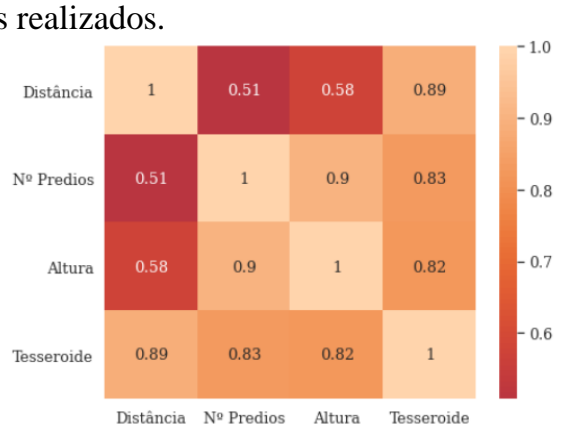

(c)

Fonte: Os autores (2021).

Assim, ficou evidenciado a existência de linearidade entre as variáveis distância, quantidade de prédios e altura dos prédios no cálculo do potencial a partir dos elementos de massa tesseroide (Figura 9.a), prisma (Figura 9.b) e massa pontual (Figura 9.c).

Após as análises envolvendo o potencial gravitacional foi estimado o valor da anomalia de gravidade associada à topografia a partir das massas das edificações. Os valores de $V$ foram empregados nesse cálculo $(\Delta \mathrm{g})$ descrito na Eq. (2). Na Tabela $4, \Delta \mathrm{g}$ Solo refere-se ao valor médio da anomalia de gravidade referente ao nível do solo para cada experimento. Neste caso, para sua obtenção utilizou-se os valores de altitude referentes ao MDT e valor da densidade $(\rho)$ igual a $2670 \mathrm{~kg} / \mathrm{m}^{3}$. Nesta tabela também é apresentado o valor médio da anomalia de gravidade associado à topografia e as massas das edificações ( $\Delta \mathrm{g}$ Prédios). Entretanto, para sua obtenção fez-se uso do modelo de densidades descrito na Tabela 1 e dos valores das alturas dos prédios medidos em relação ao MDS.

Tabela 4 - Valores do efeito topográfico das massas no cálculo da anomalia de gravidade.

\begin{tabular}{|c|c|c|c|c|}
\hline Experimento & $\Delta \mathrm{g}$ Solo $(\mathrm{mGal})$ & $\sigma \Delta \mathrm{g}$ Solo (mGal) & $\Delta \mathrm{g}$ Edif (mGal) & $\sigma \Delta \mathrm{g}$ Edif (mGal) \\
\hline $01 \mathrm{~km}$ & 7,113 & 0,026 & 0,969 & 0,278 \\
\hline $02 \mathrm{~km}$ & 7,067 & 0,068 & 0,877 & 0,264 \\
\hline $05 \mathrm{~km}$ & 6,970 & 0,161 & 0,788 & 0,246 \\
\hline $10 \mathrm{~km}$ & 6,734 & 0,523 & 0,575 & 0,325 \\
\hline $20 \mathrm{~km}$ & 6,650 & 0,753 & 0,460 & 0,396 \\
\hline Média & 6,907 & 0,306 & 0,734 & 0,302 \\
\hline \multicolumn{4}{|c|}{ Relação entre $\Delta \mathrm{g}$ Edificações $/ \Delta \mathrm{g}$ Solo } & $10,62 \%$ \\
\hline
\end{tabular}

Fonte: Os autores (2021).

A partir dos valores descritos na Tabela 4 e Figura 10, chegou-se ao resultado que a influência das massas das edificações associadas a topografia representaram 10,62\% do valor da anomalia de gravidade em comparação com o seu correspondente em relação ao solo. Na Tabela 4 também consta o valor do desviopadrão da anomalia de gravidade calculado a partir das massas das edificações e em relação ao solo $(\sigma \Delta \mathrm{g}$ Edif e $\sigma \Delta$ Solo). Os maiores valores de desvio-padrão ocorreram nos experimentos com maior número de 
edificações, nesse caso os experimentos com as distâncias de 10 e 20 km.

Figura 10 - Comparação do efeito topográfico das edificações em relação ao solo.

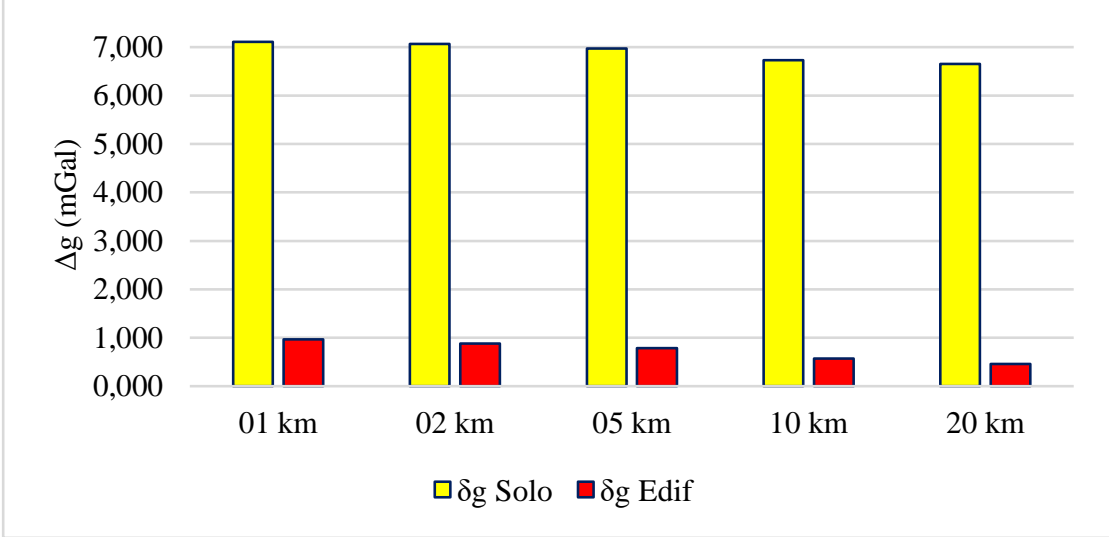

Fonte: Os autores (2021).

Esse valor segue a literatura, no caso Schwarz (1984) onde o autor descreve que entre 2 e $34 \%$ dos espectros relacionados ao campo de gravidade associados ao cálculo do geoide estão contidos nas altas frequências relacionadas a MDE de alta resolução. Embora não seja o foco principal desse estudo, e fazendo uma comparação a partir dos resultados do estudo de Tziavos, Vergos e Grigoriadis (2010), estima-se os dados do presente estudo podem gerar uma influência de 3,85 e 6,49 cm no valor do cálculo do geoide em regiões com uma grande quantidade de edificações como por exemplo grandes cidades.

\section{CONSIDERAÇÕES FINAIS}

O presente estudo teve como objetivo principal estimar o valor do potencial gravitacional de massas de edificações, juntamente com a anomalia de gravidade associada à topografia na cidade de Porto Alegre RS, usando um modelo digital de elevação (MDE) de alta resolução, oriundo de dados LiDAR. Esse tipo de modelagem foi realizada utilizando a modelagem RTM (residual terrain modelling), empregando os elementos de massa tesseroide, prima e massa pontual, em que cada elemento visou a modelar uma base de dados vetorial com mais de 190 mil edificações.

Estudos que abordam esse tipo de modelagem, como Heck e Seitz (2007) e Wild-Pfeifer (2008), geralmente utilizam os valores de altitudes e densidade como constantes. Nesse estudo, procurou-se variar o tipo de parâmetro e estimar o valor do potencial e da anomalia de gravidade relacionada com as massas das edificações. Os resultados dos experimentos desenvolvidos nesse trabalho apresentaram valores coerentes, dentro da proporção das variáveis empregadas no trabalho de Heck e Seitz (2007), no qual as maiores variações do valor do potencial calculado foram a partir de distâncias acima de nove quilômetros.

Nesse contexto, a utilização da técnica de RTM com o emprego de um modelo digital de elevações (MDE) de alta resolução, a partir de dados LiDAR, foi fundamental para cálculo do valor do potencial gravitacional e do cálculo da anomalia de gravidade relacionada à topografia. A partir das alturas derivadas do MDE, estimou-se um modelo de densidade para cada edificação.

Como a densidade é um dos principais parâmetros na integral de Newton, também foram avaliadas as variáveis que geraram o modelo de distribuição de massas, como a altura dos prédios, a quantidade de edificações e também a distância do ponto de cálculo até o centro de massa de cada prédio. A partir de uma análise de correlação, verificou-se que essas variáveis possuem uma relação linear com o valor do potencial obtido em cada modelo de massa analisado.

Notou-se também que os valores do potencial utilizando os modelos de massa tesseoide, prisma e massa pontual apresentaram uma variação muito pequena dentro de cada experimento (no caso distâncias de 01, 02, 05, 10 e $20 \mathrm{~km}$ ). Por meio de uma análise variância (ANOVA), estimou-se que, para esse estudo, aos valores do potencial gravitacional calculado não apresentam diferença significativa. Acredita-se que isso ocorreu em função da limitação da distância máxima adotada nesse estudo, que foi de $20 \mathrm{~km}$. 
A partir dos resultados obtidos nesse trabalho, estima-se que a principal contribuição desse estudo foi calcular o valor do potencial gravitacional juntamente com a anomalia de gravidade associada à topografia relacionada às massas de edificações, utilizando a modelagem RTM. Estimou-se que o valor da anomalia de gravidade associada às massas das edificações apresentaram resultados de magnitude de $10,62 \%$ em relação ao seu correspondente associado à superfície terrestre. Assim, baseado nesses resultados, acredita-se que esse tipo de análise deve ser levado em consideração no cálculo de modelos geoidais locais de regiões com grande densidade de edificações (por exemplo, grandes cidades). Obteve-se uma grande otimização no tempo de processamento ao trabalhar com uma base vetorial num ambiente integrado de desenvolvimento (IDE), utilizando o Google Colab. Essa escolha possibilitou a utilização dos MDEs de alta resolução, gerados a partir dos dados LiDAR. Caso fosse utilizada uma base de dados matricial, seria necessário o uso de um computador com alta capacidade de processamento.

\section{Agradecimentos}

Agradecimento ao Conselho Nacional de Desenvolvimento Científico e Tecnológico (CNPq) pela concessão da Bolsa de Produtividade em Pesquisa com n ${ }^{\circ}$ de processo 303046/2017, para o segundo autor.

\section{Contribuição dos Autores}

O autor principal, Rodrigo da Silva Ferraz, doutorando do Programa de Pós-Graduação em Sensoriamento Remoto, foi o responsável por separar e filtrar a base vetorial que serviu de apoio para esse estudo. O referido autor também implementou os cálculos na linguagem de programação Python, fez a pesquisa e fundamentação teórica e escreveu o conteúdo do texto dessa pesquisa. O Prof. Dr. Sérgio Florêncio de Souza, do Programa de Pós-Graduação em Sensoriamento Remoto da UFRGS, foi o responsável pelo tema do trabalho e pela correção e revisão do texto.

\section{Conflitos de Interesse}

Os autores declaram que não há conflitos de interesse.

\section{Referências}

DENG, X. L.; SHEN, W. B. Topographic effects up to gravitational curvatures of tesseroids: A case study in China. Studia Geophysica et Geodaetica, v. 63, n. 3, p. 345-366, 2019. DOI. 10.1007/s11200-018-07724.

DIRETORIA DE SERVIÇO GEOGRÁFICO (DSG). Banco de Dados Geográfico do Exército. Disponível em: < https://bdgex.eb.mil.br/mediador/>. Acesso em: 10 jul. 2020.

FORSBERG, R. Study of terrain reductions, density anomalies and geophysical inversion methods in gravity-field modeling. Technical report. Ohio State Univ. Research Foundation, Columbus (USA), 1984.

GEMAEL, C. Introdução à geodésia física. Curitiba: Editora da UFPR, 1999.

GUIMARÃES, G. DO N.; BLITZKOW, D. Problema de valor de contorno da Geodésia: uma abordagem conceitual. Boletim de Ciências Geodésicas, v. 17, n. 4, p. 607-624, 2011. DOI. 10.1590/s198221702011000400007.

GROMBEIN, T.; SEITZ, K.; HECK, B. Optimized formulas for the gravitational field of a tesseroid. Journal of Geodesy, v. 87, n. 7, p. 645-660, 2013. DOI. 10.1007/s00190-013-0636-1.

HECK, B.; SEITZ, K. A comparison of the tesseroid, prism and point-mass approaches for mass reductions in gravity field modelling. Journal of Geodesy, v. 81, n. 2, p. 121-136, 2007. DOI. 10.1007/s00190-0060094-0 
HEISKANEN, W. A.; MORITZ, H. Physical geodesy. San Francisco: WH Freeman and Company, 1967.

HIRT, C.; BUCHA, B.; YANG, M.; KUHN, M. A numerical study of residual terrain modelling (RTM) techniques and the harmonic correction using ultra-high-degree spectral gravity modelling. Journal of Geodesy, v. 93, n. 9, p. 1469-1486, 2019. DOI. 10.1007/s00190-019-01261-x.

KUHN, M.; HIRT, C. Topographic gravitational potential up to second-order derivatives: an examination of approximation errors caused by rock-equivalent topography (RET). Journal of Geodesy, v. 90, n. 9, p. 883-902, 2016. DOI. 10.1007/s00190-016-0917-6.

LI, Z.; HAO, T.; XU, Y.; XU, Y. An efficient and adaptive approach for modeling gravity effects in spherical coordinates. Journal of Applied Geophysics, v. 73, n. 3, p. 221-231, 2011. DOI. 10.1016/j.jappgeo.2011.01.004.

LIN, M.; DENKER, H.; MÜLLER, J. Gravity Field Modeling Using Tesseroids with Variable Density in the Vertical Direction. Surveys in Geophysics, p. 1-43, 2020. DOI. 10.1007/s10712-020-09585-6.

MAROTTA, G.; ALMEIDA, Y.; CHUERUBIM, M. L. Análise da Influência do Valor de Densidade na Estimativa do Modelo Geoidal Local para o Distrito Federal, Brasil. Revista Brasileira de Cartografia, v. 71, n. 4, p. 1089-1113, 2019. DOI. 0000-0003-1073-0683 .

MAROTTA, A. M.; SEITZ, K.; BARZAGHI, R.; GROMBEIN, T.; HECK, B. Comparison of two different approaches for computing the gravitational effect of a tesseroid. Studia Geophysica et Geodaetica, v. 63, n. 3, p. 321-344, 2019. DOI: 10.1007/s11200-018-0454-2.

MIKHAIL, E. M.; BETHEL, J. S.; MCGLONE, J. C. Introduction to modern photogrammetry. New York, 2001.

MOnTGomery, D. C.; RUnGer, G. C.; CAlAdO, V. Estatística Aplicada E Probabilidade Para Engenheiros. Rio de Janeiro: Grupo Gen-LTC, 2000.

NAGY, D.; PAPP, G.; BENEDEK, J. The gravitational potential and its derivatives for the prism. Journal of Geodesy, v. 74, n. 7-8, p. 552-560, 2000. DOI. 10.1007/s001900000116.

OMANG, O. C. D.; FORSBERG, R. How to handle topography in practical geoid determination: three examples. Journal of Geodesy, v. 74, n. 6, p. 458-466, 2000. DOI. 10.1007/s001900000107.

PREFEITURA MUNICIPAL DE PORTO ALEGRE (PMPA). Secretaria Municipal de Urbanismo. Viva o Centro. Disponível em: < http://www2.portoalegre.rs.gov.br/vivaocentro/default.php?p_secao=18>. Acesso em: 10 jul. 2020.

REXER, M.; HIRT, C.; BUCHA, B.; HOLMES, S. Solution to the spectral filter problem of residual terrain modelling (RTM). Journal of Geodesy, v. 92, n. 6, p. 675-690, 2018. DOI. 10.1007/s00190-017-1086-y.

SARASWATI, A. T.; CATTIN, R.; MAZZOTTI, S.; CADIO, C. New analytical solution and associated software for computing full-tensor gravitational field due to irregularly shaped bodies. Journal of Geodesy, v. 93, n. 12, p. 2481-2497, 2019. DOI. 10.1007/s00190-019-01309-y.

SHAN, J.; TOTH, C. K. Topographic laser ranging and scanning: principles and processing. Boca Raton: CRC Press, 2008.

SCHWARZ, K. P. Data types and their spectral properties. In: SCHARTZ K.P. (Ed.). Local gravity field approximation. International Summer School (BSS). Beijing, 1984. p. 1-66.

SÜNKEL, H. GBVP — Classical solutions and implementation. In: SANSÓ, F.; RUMMEL, R. (Ed.). Geodetic Boundary Value Problems in View of the One Centimeter Geoid. Lecture Notes in Earth Sciences. Berlin, Heidelberg: Springer, 1997. p. 219-237.

VARGA, M.; GRGIĆ, M.; BJELOTOMIĆ ORŠULIĆ; O.; BAŠIĆ, T. Influence of digital elevation model resolution on gravimetric terrain correction over a study-area of Croatia. Geofizika, v. 36, n. 1, p. 17-32, 2019. DOI. 10.15233/gfz.2019.36.1.

WILD-PFEIFFER, F. A comparison of different mass elements for use in gravity gradiometry. Journal of Geodesy, v. 82, n. 10, p. 637-653, 2008. DOI. 10.1007/s00190-008-0219-8.

YAHAYA, S. I.; EL AZZAB, D. High-resolution residual terrain model and terrain corrections for gravity 
field modelling and geoid computation in Niger Republic. Geodesy and Cartography, v. 44, n. 3, p. 8999, 2018. DOI. 10.3846/gac.2018.3787.

YANG, M.; HIRT, C.; PAIL, R. TGF: A New MATLAB-Based Software for Terrain-Related Gravity Field Calculations. Remote Sensing, v. 12, n. 7, p. 1063, 2020. DOI. 10.3390/rs12071063.

THE SEATTLE TIMES. What does a house weigh? Some mental heavy lifting., 19. dez. 2004. Disponível em <https://archive.seattletimes.com/archive/?date=20041219\&slug=homehay19>. Acesso em: 12/7/2020.

TZIAVOS, I. N.; VERGOS, G. S.; GRIGORIADIS, V. N. Investigation of topographic reductions and aliasing effects on gravity and the geoid over Greece based on various digital terrain models. Surveys in geophysics, v. 31, n. 1, p. 23-67, 2010. DOI. 10.1007/s10712-009-9085-z.

TZIAVOS, I. N.; SIDERIS, M. G. Topographic Reductions in Gravity and Geoid Modeling. In: SANSÒ, F.; SIDERIS, M. G. (Ed.). Geoid Determination: Theory and Methods. Lecture Notes in Earth System Sciences. Berlin, Heidelberg: Springer, 2013. p. 337-400.

TSOULIS, D.; NOVÁK, P.; KADLEC, M. Evaluation of precise terrain effects using high-resolution digital elevation models. Journal of Geophysical Research: Solid Earth, v. 114, n. B2, p. B02404, 2009. DOI. 10.1029/2008JB005639

VOSSELMAN, G.; MAAS, H. G. Airborne and terrestrial laser scanning. Boca Raton: CRC Press, 2010.

ZANARDI, R. P.; SCHNEIDER, A. H.; SALOMONI, T. R.; SALOMONI, C. S.; REISS, M. L. L. (2013). Validação da qualidade do perfilamento a LASER aerotransportado da cidade de Porto Alegre. In: SIMPÓSIO BRASILEIRO DE SENSORIAMENTO REMOTO, 16., 2013, Foz do Iguaçu. Anais...São José dos Campos: INPE, 2013. p. 6178-6184.

\section{Biografia do autor principal}

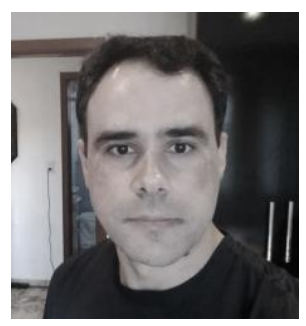

Rodrigo da Silva Ferraz, autor principal, nascimento em Xanxerê-SC em 14 de abril de 1980. Graduado em Engenharia Cartográfica pela Universidade Federal do Rio Grande do Sul (UFRGS) no ano de 2014 e Mestre em Sensoriamento Remoto pela UFRGS em 2017. Experiência profissional na área de Geoprocessamento e Topografia desde o ano de 2002 trabalhando pelo Exército Brasileiro no $9^{\circ}$ Batalhão de Engenharia de Construção em Cuiabá-MT, de 2002 até 2006, e pelo $1^{\circ}$ Centro de Geoinformação em Porto Alegre - RS, de 2007 até hoje. 